\title{
Ureia como fonte de nitrogênio na fisiologia e crescimento inicial de Guazuma ulmifolia Lam. (Malvaceae)
}

\author{
Urea as a source of nitrogen in physiology and initial growth of Guazuma \\ ulmifolia Lam. (Malvaceae)
}

\author{
Patrícia Oliveira da Silva ${ }^{\mathrm{I}}$, Leandro Carlos ${ }^{\mathrm{II}}$, \\ Andréia Mendes da Costa ${ }^{\mathrm{III}}$, Jorciane Souza Dias ${ }^{\mathrm{IV}}$, \\ Vitor Martins Venezianov ${ }^{\mathrm{V}}$, Carlos Ribeiro Rodrigues ${ }^{\mathrm{vI}}$
}

\begin{abstract}
Resumo
O objetivo deste estudo foi determinar de que forma as mudas de Guazuma ulmifolia respondem a doses nitrogenadas, tendo como fonte de nitrogênio $(\mathrm{N})$ a ureia. O cultivo das mudas ocorreu em delineamento inteiramente ao acaso, as quais foram submetidas a cinco doses $\left(0,50,100,150\right.$ e $\left.200 \mathrm{mg} \mathrm{dm}^{-3}\right)$ de N. Aos 120 dias de cultivo foram realizadas avaliações biométricas, fisiológicas, acúmulo de biomassa, razões alométricas, índice de qualidade de Dickson (IQD), teor, acúmulo e eficiência do uso de N (EUN) na parte aérea das mudas. Os dados foram submetidos a análise de variância e regressão. Além disso, o coeficiente de correlação de Pearson também foi analisado. As doses de $\mathrm{N}$ promoveram significativamente $(\mathrm{p} \leq 0,05)$ o aumento do teor de $\mathrm{N}$ na parte aérea das mudas. Por outro lado, registrou-se a redução significativa do acúmulo de $\mathrm{N}$, de variáveis fisiológicas, crescimento, biomassa, IQD e EUN, que culminou em correlação negativa com o fornecimento crescente de $\mathrm{N}$. Doses de $\mathrm{N}$ muito elevadas, tendo como fonte a ureia, foram prejudiciais para as mudas de Guazuma ulmifolia. As doses entre 20 e $38,8 \mathrm{mg} \mathrm{dm}^{-3} \mathrm{de} \mathrm{N}$, de modo geral, foram as que promoveram os melhores resultados.
\end{abstract}

Palavras-chave: Mutamba; Adubação nitrogenada; Variáveis fisiológicas

\begin{abstract}
The aim of this study was to determine how Guazuma ulmifolia seedlings respond to nitrogen doses, with urea as a nitrogen source $(\mathrm{N})$. The seedlings were cultivated in a completely randomized design, which were subjected to five doses $\left(0,50,100,150\right.$ and $\left.200 \mathrm{mg} \mathrm{dm}^{-3}\right)$ of N. At 120 days of cultivation, biometric, physiological, biomass accumulation, allometric ratios, Dickson's quality index (IQD), content, accumulation and N efficiency use (NUE) in the aerial part of the seedlings. The data were submitted to analysis of variance and regression. In addition, Pearson's correlation coefficient was also analyzed. The $\mathrm{N}$ doses significantly promoted $(\mathrm{p} \leq 0.05)$ the increase in the $\mathrm{N}$ content in the aerial part of the seedlings. On the other hand, there was a significant reduction in the $\mathrm{N}$ accumulation, of physiological variables, growth, biomass, IQD and NUE, which resulted in a negative correlation with the increasing $\mathrm{N}$ supply. Very high $\mathrm{N}$ doses, having as source the urea, were harmful to the seedlings of Guazuma ulmifolia. The doses between 20 and $38.8 \mathrm{mg} \mathrm{dm}^{-3}$ of $\mathrm{N}$, in general, were those that promoted the best results.
\end{abstract}

Keywords: Mutamba; Nitrogen fertilization; Physiological variables

Bióloga, Ma., Programa de Pós-graduação em Ciências Agrárias-Agronomia, Instituto Federal Goiano, campus Rio Verde, Rod. Sul Goiana, Km 01, CEP 75901-970, Rio Verde (GO), Brasil. patriciasilvaifgoiano@gmail.com (ORCID: 0000-0003-2242-320X)

Engenheiro Florestal, Dr., Professor do Instituto Federal Goiano, campus Rio Verde, Rod. Sul Goiana, Km 01, CEP 75901-970, Rio Verde (GO), Brasil. leandro.carlos@ifgoiano.edu.br (ORCID: 0000-0003-1736-6079)

III Engenheira Agrícola, Ma., Programa de Pós-graduação em Ciências Agrárias-Agronomia, Instituto Federal Goiano, campus Rio Verde, Rod. Sul Goiana, Km 01, CEP 75901-970, Rio Verde (GO), Brasil. andreiamendes226@gmail.com (ORCID: 0000-0003-0395-6190)

Iv Engenheira Ambiental, Instituto Federal Goiano, campus Rio Verde, Rod. Sul Goiana, Km 01, CEP 75901-970, Rio Verde (GO), Brasil. jorciane souza@hotmail.com (ORCID: 0000-0001-8412-4737)

Engenheiro Agrônomo, Me., Instituto Federal Goiano, campus Rio Verde, Rod. Sul Goiana, Km 01, CEP 75901-970, Rio Verde (GO), Brasil. vv_ martins@hotmail.com (ORCID: 0000-0002-6488-2300)

v1 Engenheiro Agrônomo, Dr., Professor do Departamento de Agronomia, Instituto Federal Goiano, campus Rio Verde, Rod. Sul Goiana, Km 01, CEP 75901-970, Rio Verde (GO), Brasil. carlos.rodrigues@ifgoiano.edu.br (ORCID: 0000-0001-7533-1179) 


\section{Introdução}

O nitrogênio $(\mathrm{N})$ é um elemento essencial para o crescimento e desenvolvimento das plantas por compor moléculas de ácidos nucleicos, aminoácidos, proteínas e clorofilas (MORAES et al., 2017). E entre as principais fontes de $\mathrm{N}$ está a ureia, considerada o fertilizante sólido nitrogenado mais utilizado no mundo (SOUZA, 2018), por causa da maior concentração de N por quilograma do produto (GALINDO et al., 2018). No entanto, a ureia não é absorvida diretamente pelas plantas, sendo necessário a conversão em amônio ou nitrato. Ao ser aplicada ao solo, a ureia passa por hidrolização pela ação da urease, resultando em carbonato de amônio, que se decompõe, originando o amônio, bicarbonato e hidroxila. Parte desse amônio pode ser absorvido pelas plantas ou convertido em amônia, e esta pode se perder na atmosfera (ROCHETTE et al., 2009), ou ser convertida em nitrato por microrganismos presentes no solo, nesse caso bactérias nitrificantes.

$\mathrm{O} \mathrm{N}$, além de ser o constituinte primário de várias moléculas está envolvido na sinalização que influencia grande número de processos vegetais, incluindo o crescimento de raízes laterais, resistência a estresses bióticos e abióticos, regulação da germinação de sementes e mediação de sinalização hormonal (WANG; HSU; TSAY, 2012). A ausência desse nutriente afeta a taxa fotossintética, potencial osmótico e o crescimento da parte aérea (MAGADLELA et al., 2014). Em contrapartida, estimula o crescimento do sistema radicular, a fim de obter acesso a maior volume de solo em busca de mais N (RAJAN; ANANDHAN, 2016), para garantir o crescimento, manutenção do turgor celular e absorção de água e outros nutrientes para atender às necessidades da planta.

Apesar da importância da adubação nitrogenada, não se sabe como se comporta as mudas de algumas espécies sob esse tipo de adubação. Esse é o caso de Guazuma ulmifolia Lam. (Malvaceae), popularmente conhecida como mutamba ou guásimo, uma vez que pesquisas dessa natureza com tal espécie são escassas. A espécie é amplamente encontrada no México, América Central e do Sul, sendo que no território brasileiro ocorre em todos os domínios fitogeográficos (COLLI-SILVA, 2019). É uma planta extremamente importante por apresentar princípios ativos utilizados no combate a alguns parasitas como Leishmania brasiliensis, Leishmania infantum e Trypanossoma cruzi (CALIXTO JÚNIOR et al., 2016). E, recentemente tem sido utilizada como tratamento alternativo contra o vírus do HIV no Brasil (GOUVEIA, 2018) e Venezuela (PORTADORES..., 2018; SINGER, 2018; MACEDO, 2019).

Considerando o fato de que o $\mathrm{N}$ é o nutriente mais exigido e, portanto, absorvido em maior quantidade pelas plantas (FERNANDES et al., 2019), levantou-se a hípotese de que o crescimento inicial de Guazuma ulmifolia possa ser potencializado pela adubação nitrogenada. $\mathrm{E}$, para testar tal hípotese, este estudo teve o objetivo de avaliar o comportamento das mudas de Guazuma ulmifolia submetidas a diferentes doses de N, tendo como fonte a ureia.

\section{Material e métodos}

Este estudo foi conduzido em casa de vegetação do Instituto Federal Goiano, campus Rio Verde, sob delineamento experimental inteiramente ao acaso, composto por cinco tratamentos $\left(0,50,100,150\right.$ e $200 \mathrm{mg} \mathrm{dm}^{-3}$ de N) e quatro repetições para cada dose. O solo utilizado como substrato foi um LATOSSOLO VERMELHO Distrófico (SANTOS et al., 2018), coletado na camada de 0,0 a 0,20 metros de profundidade, e em condição natural apresentou as seguintes características: $\mathrm{pH}\left(\mathrm{CaC}_{2}\right)$ de 5,1;0,05 $\mathrm{cmol}_{c} \mathrm{dm}^{-3}$ de alumínio; $1,06 \mathrm{cmol} \mathrm{dm}^{-3}$ de cálcio; 0,49 $\mathrm{cmol}_{\mathrm{c}} \mathrm{dm}^{-3}$ de magnésio; $2,6 \mathrm{mg} \mathrm{dm}^{-3}$ de fósforo; $128 \mathrm{mg} \mathrm{dm}^{-3}$ de potássio; $41,3 \mathrm{~g} \mathrm{dm}^{-3}$ de matéria orgânica, 47 \% de saturação de bases e 48,40 e 12\% de argila, areia e silte, respectivamente.

A saturação de bases foi elevada a $60 \%$ (RAIJ, 1997) com os corretivos carbonato de cálcio e carbonato de magnésio na proporção de 4:1. A ureia convencional granular $\left(\mathrm{CO}\left(\mathrm{NH}_{2}\right)_{2}\right.$, 
foi utilizada como fonte de $\mathrm{N}$ e aplicada 10 dias antes da adubação básica e 15 dias antes da semeadura. A adubação básica $\left(\mathrm{mg} \mathrm{dm}^{-3}\right)$ foi realizada 30 dias ápos a realização da calagem com o fornecimento de: 300 de P, 150 de K, 40 de S, 1,33 de Cu, 0,81 de B e 4 de $\mathrm{Zn}$. As fontes utilizadas foram: superfosfato triplo, sulfato de potássio e cloreto de potássio, sulfato de cobre, ácido bórico e sulfato de zinco, respectivamente (CARLOS et al., 2015).

As sementes de Guazuma ulmifolia foram coletadas em 10 matrizes adultas localizadas na Universidade Federal de Lavras. A dormência foi superada com água quente a $70^{\circ} \mathrm{C}$ (NUNES et al., 2006) e a semeadura ocorreu 45 dias após a correção da acidez do solo. Os recipientes utilizados foram vasos de quatro litros com fundo sem furos. A irrigação foi realizada diariamente mantendo a terra com $60 \%$ da capacidade de campo através da pesagem dos vasos com as plantas, irrigando diretamente o solo. E, aos 120 dias após a semeadura, deu-se início as avaliações.

As avaliações biométricas consistiram da altura de planta $(\mathrm{H})$ obtida por meio de régua milimetrada enquanto o diâmetro do coleto (DC) foi obtido com paquímetro digital. Também foi contabilizado o número de folhas totalmente expandidas (NF).

As análises de trocas gasosas foram determinadas em uma das folhas do segundo par, a partir do ápice da planta, entre 8 e 11 horas da manhã, sendo as variáveis avaliadas: taxa fotossintética $\left.\left[A, \mu \mathrm{mol}\left(\mathrm{CO}_{2}\right) \mathrm{m}^{-2} \mathrm{~s}^{-1}\right)\right]$ e transpiratória $\left[E, \mathrm{mmol}\left(\mathrm{H}_{2} \mathrm{O}\right) \mathrm{m}^{-2} \mathrm{~s}^{-1}\right.$, condutância estomática $\left[g\right.$, mol $\left.\left(\mathrm{H}_{2} \mathrm{O}\right) \mathrm{m}^{-2} \mathrm{~s}^{-1}\right]$, concentração interna de $\mathrm{CO}_{2}\left(\mathrm{Ci}, \mu \mathrm{mol} \mathrm{mol}{ }^{-1}\right)$, concentração externa de $\mathrm{CO}_{2}\left(\mathrm{Ca}, \mu \mathrm{mol} \mathrm{mol}^{-1}\right)$, razão entre a concentração interna e externa de $\mathrm{CO}_{2}(\mathrm{Ci} / \mathrm{Ca}$, $\mu \mathrm{mol} \mathrm{mol}{ }^{-1}$ ), eficiência do fotossistema II (ØPS2) e taxa de transporte de elétrons (ETR, $\mu \mathrm{mol}$ $\mathrm{m}^{-2} \mathrm{~s}^{-1}$ ), realizadas por meio do analisador de gases infravermelho portátil (Modelo Li-6800XT, Nebraska, EUA).

Ao fim do cultivo das mudas, as plantas foram retiradas dos vasos e após a limpeza, realizou-se a separação das folhas, caules e raízes, e, posteriormente, acondicionados em estufa de circulação de ar forçado a $65^{\circ} \mathrm{C}$ até obter a massa constante. E, por fim, em balança digital foram obtidos a massa seca foliar (MSF), massa seca caulinar (MSC), massa seca radicular (MSR). Com esses dados, calculou-se a massa seca total (MST) para calcular as razões alométricas: razão de massa foliar (MSF/MST, $\mathrm{g} \mathrm{g}^{-1}$ ), razão de massa caulinar (MSC/MST, $\mathrm{g} \mathrm{g}^{-1}$ ) e razão de massa radicular (MSR/MST, $\mathrm{g} \mathrm{g}^{-1}$ ). Mediante aos dados de crescimento e biomassa calculou-se o Índice de qualidade de Dickson (DICKSON; LEAF; HOSNER, 1960). Com registros de imagens fotográficas das folhas de cada unidade experimental, mensurou-se com o software Image $J{ }^{\circledR}$ (HIN, Bethesda, Maryland, EUA) a área foliar (AF). E com os dados de área foliar e massa seca foliar, calculou-se a área foliar específica (AFE) através da fórmula: AFE = AF/MSF.

$\mathrm{O}$ teor de $\mathrm{N}$ da parte área das mudas foi determinado utilizando metodologia de Kjeldahl, em que as amostras foram secas em estuda e moídas em moinho tipo Wiley em malha fina $(2 \mathrm{~mm})$, após a pesagem, passaram por digestão em mistura catalítica, composta de sulfato de potássio e sulfato de cobre na proporção de 10:1, ácido sulfúrico e peróxido de hidrogênio, e em seguida submetida a destilação com liberação da amônia em que é fixada em solução de ácido bórico e posteriormente titulada com solução padrão de ácido clorídrico. $\mathrm{O}$ acúmulo de $\mathrm{N}$ da parte aérea $(\mathrm{AcN})$ foi obtido através da multiplicação do teor de $\mathrm{N}$ com a biomassa da parte aérea das mudas. $\mathrm{E}$ a eficiência de uso de nitrogênio $(\mathrm{EUN})$ da parte aérea foi calculada através da fórmula: EUN = $(\mathrm{MSPA})^{2} /(\mathrm{AcN})$ (SIDDIQI; GLASS, 1981).

Os dados experimentais foram analisados de acordo com os pressupostos básicos de normalidade e homogeneidade, e posteriormente submetidos a análise de variância pelo teste $\mathrm{F}$ e análise de regressão. A escolha do modelo ocorreu em função da significância de cada equação. O coeficiente de correlação linear de Pearson também foi calculado envolvendo as variáveis entre si. Para a realização das análises estatísticas, utilizou-se os softwares SISVAR (FERREIRA, 2014) e BIOESTAT (AYRES et al., 2007), respectivamente, a $5 \%$ de probabilidade de erro. 


\section{Resultados e discussão}

$\mathrm{O}$ fornecimento crescente de $\mathrm{N}$ por meio da adubação com ureia promoveu aumento significativo $(\mathrm{p} \leq 0,05)$ do teor de $\mathrm{N}$ na parte aérea das mudas (Figura $1 \mathrm{~A})$. Em contrapartida, houve a redução significativa do acúmulo de $\mathrm{N}$ na parte aérea, eficiência do uso do nitrogênio na parte aérea, eficiência do fotossistema II, taxa de transporte de elétrons e taxa fotossintética das mudas de Guazuma ulmifolia (Figura 1B, C, D, E e F), sendo as doses de máxima eficiência 26,31; 5,0; 38,$88 ; 18,75$ e $25,64 \mathrm{mg} \mathrm{dm}^{-3}$ de $\mathrm{N}$, respectivamente. A condutância estomática $\left(0,238 \mathrm{~mol}\left(\mathrm{H}_{2} \mathrm{O}\right)\right.$ $\left.\mathrm{m}^{-2} \mathrm{~s}^{-1}\right)$, taxa transpiratória $\left(0,0028 \mathrm{mmol}\left(\mathrm{H}_{2} \mathrm{O}\right) \mathrm{m}^{-2} \mathrm{~s}^{-1}\right)$, concentração interna de $\mathrm{CO}_{2}(270,13 \mu \mathrm{mol}$ $\left.\mathrm{mol}^{-1}\right)$, e razão da concentração interna e externa de $\mathrm{CO}_{2}\left(0,669 \mu \mathrm{mol} \mathrm{mol}^{-1}\right)$ não foram afetadas pelos tratamentos deste estudo ( $\mathrm{p}>0,05)$.

Os resultados apresentados acima mostram que as mudas de Guazuma ulmifolia apresentaram elevada sensibilidade a adubação nitrogenada por meio da ureia, pois ao mesmo tempo em que ocorreu aumento do teor houve a redução do acúmulo de $\mathrm{N}$ (efeito de concentração) na parte aérea das mudas em função dos tratamentos. Isso indica que as doses elevadas de $\mathrm{N}$ se caracterizaram como prejudicial para as mudas, já que a eficiência do uso do $\mathrm{N}$ diminuiu com o aumento de $\mathrm{N}$. A maior eficiência do uso de $\mathrm{N}$ se deu na dose de $5 \mathrm{mg} \mathrm{dm}^{-3}$ demonstrando que as mudas conseguem aproveitar pequena quantidade de $\mathrm{N}$ e maximizar seu ganho em crescimento e biomassa. No entanto, acredita-se que esses resultados não estão relacionados apenas a quantidade de $\mathrm{N}$ fornecido, mas principalmente a fonte utilizada, a ureia. Para Borges e Silva (2011) a combinação de fertilizantes nitrogenados e potássicos tendo como fonte a ureia e cloreto de potássio, exatamente como utilizados neste estudo, é prejudicial por possuírem índices salinos elevados.

Em termos fisiológicos, os tratamentos nitrogenados promoveram danos que culminaram na redução da eficiência do fotossistema II, taxa de transporte de elétrons e taxa fotossintética das mudas. Por esse motivo, tais variáveis se mostraram correlacionadas negativamente ao fornecimento crescente de N (Tabela 1). Sabe-se que em condições salinas pode ocorrer modificações na composição, funcionalidade do aparato fotossintético e no estado funcional das membranas dos tilacoides dos cloroplastos, ocasionando mudanças nas características dos sinais de fluorescência, além do rendimento quântico potencial, especialmente do fotossistema II (SILVA et al., 2011). Além dos danos fisiológicos, alguns estudos relataram que maior disponibilidade de $\mathrm{N}$ também tem promovido a limitação de outros nutrientes importantes, como potássio, cálcio e magnésio (TIAN et al., 2017; MAO et al., 2018) que pode afetar o crescimento e desenvolvimento de plantas.

A adubação nitrogenada também promoveu a redução significativa da altura, diâmetro, número de folhas, área foliar, massa seca foliar, massa seca caulinar, massa seca radicular e Índice de qualidade de Dickson das mudas de Guazuma ulmifolia (Figura 2A, B, C, D, E, F, G e H), sendo as doses de máxima eficiência 25,5; 17;25;27,7;18;20;35, $12,7 \mathrm{mg} \mathrm{dm}^{-}{ }^{3}$ de N, respectivamente. Para a área foliar específica $\left(465 \mathrm{~cm}^{2} \mathrm{~g}^{-1}\right)$, razão de massa foliar $\left(0,35 \mathrm{~g} \mathrm{~g}^{-1}\right)$, razão de massa caulinar $(0,10 \mathrm{~g}$ $\left.\mathrm{g}^{-1}\right)$ e razão de massa radicular $\left(1,8 \mathrm{~g} \mathrm{~g}^{-1}\right)$ as diferenças encontradas não foram significativas $(\mathrm{p}>0,05)$.

Com a redução das variáveis fisiológicas, por consequência, o crescimento e acúmulo de biomassa das mudas também foram afetados, variáveis estas que foram fortemente correlacionadas por apresentarem redução em função da adubação nitrogenada (Tabela 1). Em termos de produção de biomassa, esta é uma das melhores características para se avaliar a qualidade das mudas, pois mesmo sendo destrutiva, reflete a fotossíntese líquida da planta (FERNANDES et al., 2019) e está fortemente associada com a sua capacidade de sobreviver em campo. E, ao considerar os padrões de crescimento estabelecidos que consideram mudas de boa qualidade àquelas que apresentam altura entre 20 e $35 \mathrm{~cm}$ de altura (GONÇALVES et al., 2005) e diâmetro do coleto superior a quatro mm (GOMES, 2003), pode-se dizer que a doses de máxima eficiência estimada para altura e diâmetro, 25,5 e $17 \mathrm{mg} \mathrm{dm}^{-3}$ de N, respectivamente, promoveram mudas de Guazuma ulmifolia dentro desses padrões, com altura de $25,3 \mathrm{~cm}$ e diâmetro de $5,8 \mathrm{~mm}$. 
Figura 1 - (A) Teor de nitrogênio da parte aérea, (B) Acúmulo de nitrogênio na parte aérea, (C) Eficiência do uso do nitrogênio da parte aérea, (D) eficiência do fotossistema II, (E) Taxa de transporte de elétrons e (F) Taxa fotossintética das mudas de Guazuma ulmifolia Lam. (Malvaceae) em função de doses crescentes de nitrogênio ( ${ }^{*}$ significativo a p $\leq 0,05$ )

Figure 1 - (A) Shoot nitrogen content, (B) Shoot nitrogen accumulation, (C) Nitrogen use efficiency from the aerial part, (D) photosystem II efficiency, (E) Electron transport rate and (F) Photosynthetic rate of Guazuma ulmifolia Lam. (Malvaceae) seedlings in function of increasing nitrogen doses (*significant at $\mathrm{p} \leq 0.05$ )
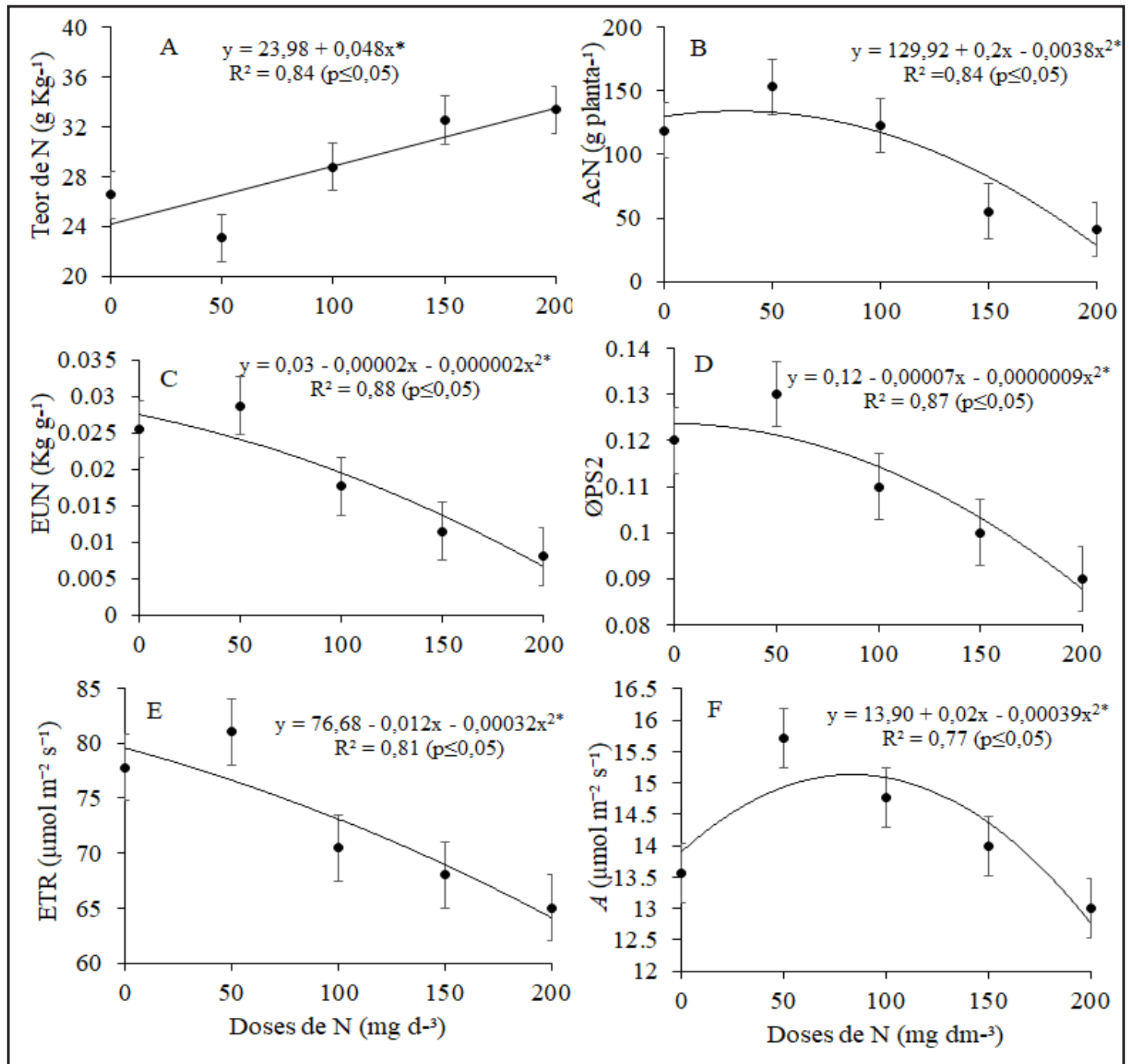

Fonte: Autores (2020)

Com a redução das variáveis biométricas e biomassa, o IQD também foi significativamente reduzido (Figura $2 \mathrm{H}$ ). E, a alta correlação encontrada (Tabela 1) entre esse índice e as variáveis supracitadas não é por acaso, uma vez que para calcular o IQD é utilizado tais variáveis. Esse índice é relevante porque é considerado um dos melhores indicadores da qualidade das mudas, pois no seu cálculo são considerados a robustez e o equilíbrio da distribuição de massa das mudas (FONSECA et al., 2002). Portanto, quanto maior o valor encontrado, maior é o padrão de qualidade, dessa forma, a dose estimada de maior eficiência foi a de 12,7 $\mathrm{mg} \mathrm{dm}^{-3}$ de N, com IQD de 5,2 . 
Figura 2 - (A) Altura, (B) Diâmetro, (C) Número de folhas, (D) Área foliar, (E) Massa seca foliar, (F) Massa seca caulinar, (G) Massa seca radicular e (H) Índice de qualidade de Dickson das mudas de Guazuma ulmifolia Lam. (Malvaceae) em função de doses crescentes de nitrogênio ( ${ }^{*}$ significativo a $\mathbf{p} \leq \mathbf{0 , 0 5}$ )

Figure 2 - (A) Height, (B) Diameter, (C) Number of leaves, (D) Leaf area,(E) Leaf dry mass,(F) Dry stem mass, (G) Root dry mass and (H) Quality index Dickson of Guazuma ulmifolia Lam.

(Malvaceae) seedlings in function of increasing nitrogen doses ( ${ }^{*}$ significant at $\mathrm{p} \leq 0.05$ )

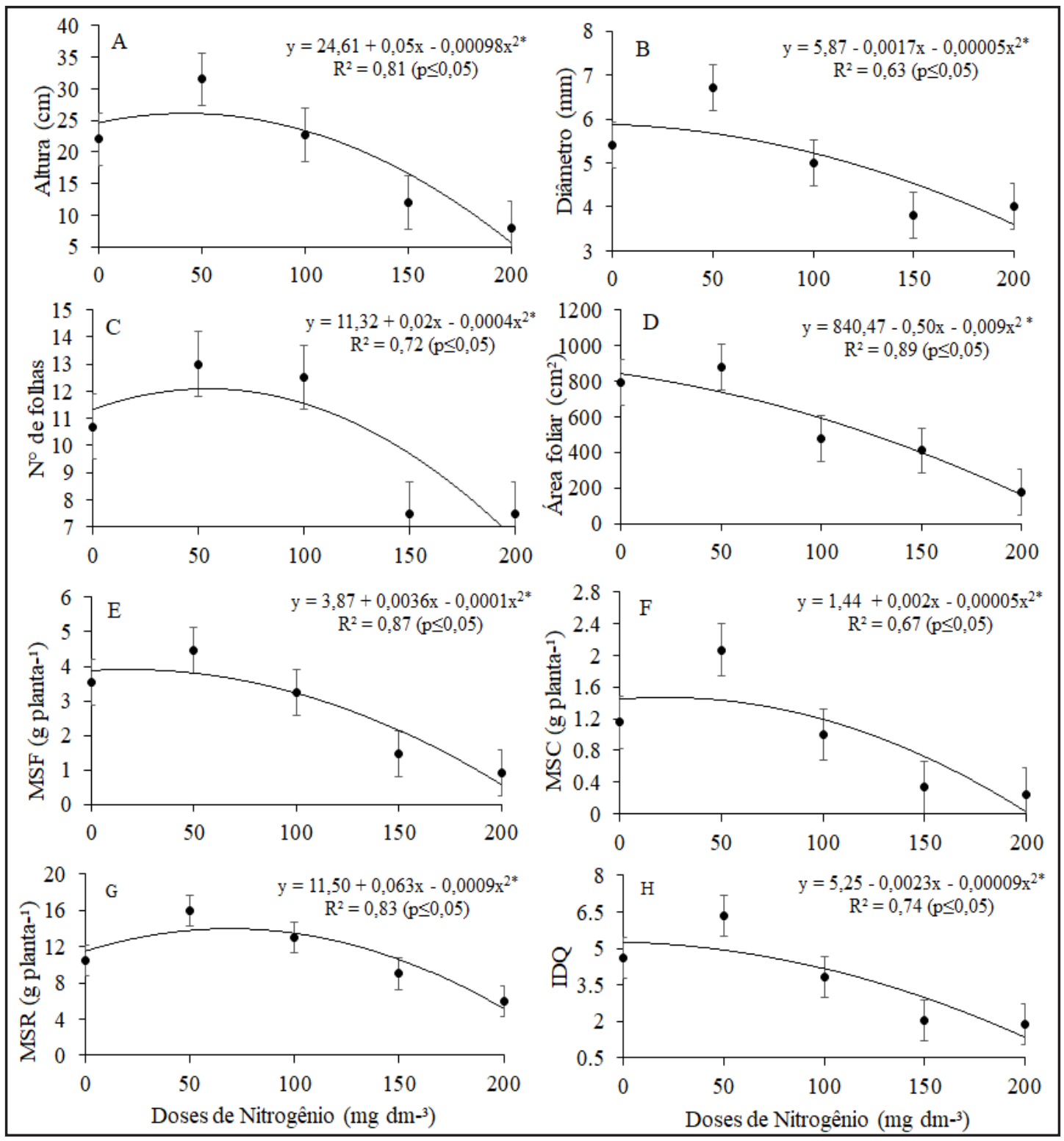

Fonte: Autores (2020)

As variáveis, principalmente biométricas e de biomassa, se mostraram altamente correlacionadas entre si. $\mathrm{O}$ teor de $\mathrm{N}$ na parte aérea das mudas se correlacionou negativamente com as variáveis biométricas, de biomassa, taxa fotossintética, eficiência do fotossistema II e a taxa de transporte de elétrons, enquanto o acúmulo de $\mathrm{N}$ se correlacionou positivamente a essas variáveis (Tabela 1). O índice de qualidade de Dickson se correlacionou positivamente com a taxa fotossintética, eficiência do fotossistema II, taxa de transporte de elétrons e acúmulo de $\mathrm{N}$ na 
parte aérea das mudas (Tabela 1). A eficiência do uso do $\mathrm{N}$ se correlacionou negativamente com a área foliar, concentração interna de $\mathrm{CO}_{2}$, condutância estomática e teor de $\mathrm{N}$. A correlação entre eficiência do uso do $\mathrm{N}$ e taxa fotossintética não foi significativa, entretanto foi significativamente positiva com as demais variáveis estudadas. Segundo Freitas et al. (2017), correlações, superiores a 0,75 , entre as variáveis indica que uma variável possibilita inferir sobre as outras, dessa forma, é valido dizer que as variáveis deste estudo estão fortemente interligadas entre si, seja negativamente ou positivamente.'

Tabela 1 - Coeficiente de correlação linear de Pearson das variáveis entre si das mudas de Guazuma ulmifolia Lam. (Malvaceae) submetidas a doses nitrogenadas. Altura, diâmetro, número de folhas, área foliar, área foliar especifica, massa seca foliar, massa seca caulinar, massa seca radicular, índice de qualidade de Dickson, taxa transpiratória, taxa fotossintética, concentração interna de $\mathrm{CO}_{2}$, condutância estomática, eficiência do fotossistema II, taxa de transporte de elétrons, teor de nitrogênio da parte área, acúmulo de $\mathrm{N}$ na parte aérea das mudas e eficiência do uso de $\mathrm{N}$ da parte aérea

Table 1 - Pearson's linear correlation coefficient of the variables among Guazuma ulmifolia Lam. (Malvaceae) seedlings submitted to nitrogen doses. Height, diameter, leaves number, leaf area, specific leaf area, leaf dry mass, stem dry matter, root dry mass, Dickson's quality index,

transpiratory rate, photosynthetic rate, internal CO2 concentration, stomatal conductance, photosystem II efficiency, rate electron transport, nitrogen content of the area, $\mathrm{N}$ accumulation in the aerial part of the seedlings and $\mathrm{N}$ efficiency use from the aerial part

\begin{tabular}{|c|c|c|c|c|c|c|c|c|c|c|c|c|c|c|c|c|c|}
\hline H & D & NF & AF & AFE & MSF & MSC & MSR & IDQ & $E$ & $A$ & $\mathrm{Ci}$ & $g s$ & ØPS2 & ETR & $\mathbf{N}$ & AcN & EUN \\
\hline H & $0,96^{*}$ & $0,96^{*}$ & $0,90^{*}$ & $-0,59^{\text {ns }}$ & $0,98^{*}$ & $0,98^{*}$ & $0,91^{*}$ & $0,97^{*}$ & $-0,82^{*}$ & $0,87^{*}$ & $-0,82^{*}$ & $-0,80^{*}$ & $0,93^{*}$ & $0,84^{*}$ & $-0,96^{*}$ & $0,98^{*}$ & $0,93^{*}$ \\
\hline D & & $0,88^{*}$ & $0,89^{*}$ & $-0,62^{\text {ns }}$ & $0,94^{*}$ & $0,98^{*}$ & $0,80^{*}$ & $0,99^{*}$ & $-0,76^{\mathrm{ns}}$ & $0,77^{\mathrm{ns}}$ & $-0,76^{\text {ns }}$ & $-0,73^{\mathrm{ns}}$ & $0,88^{*}$ & $0,78^{\text {ns }}$ & $-0,92^{*}$ & $0,94^{*}$ & $0,94^{*}$ \\
\hline $\mathrm{NF}$ & & & $0,80^{*}$ & $-0,61^{\text {ns }}$ & $0,95^{*}$ & $0,90^{*}$ & $0,90^{*}$ & $0,89^{*}$ & $-0,78^{\mathrm{ns}}$ & $0,85^{*}$ & $-0,79^{\text {ns }}$ & $-0,75^{\mathrm{ns}}$ & $0,87^{*}$ & $0,78^{\text {ns }}$ & $-0,90^{*}$ & $0,97^{*}$ & $0,82^{*}$ \\
\hline AF & & & & $-0,52^{\text {ns }}$ & $0,94^{*}$ & $0,92^{*}$ & $0,83^{*}$ & $0,93^{*}$ & $-0,93^{*}$ & $0,73^{\text {ns }}$ & $-0,93^{*}$ & $-0,92^{*}$ & $0,96^{*}$ & $0,90^{*}$ & $-0,94^{*}$ & $0,90^{*}$ & $0,98^{*}$ \\
\hline AFE & & & & & $-0,65^{\mathrm{ns}}$ & $-0,55^{\text {ns }}$ & $-0,29^{\text {ns }}$ & $-0,65^{\text {ns }}$ & $0,42^{\text {ns }}$ & $-0,29^{\text {ns }}$ & $0,43^{\text {ns }}$ & $0,23^{\text {ns }}$ & $-0,41^{\mathrm{ns}}$ & $-0,20^{\text {ns }}$ & $0,43^{\text {ns }}$ & $-0,69^{\text {ns }}$ & $-0,87^{*}$ \\
\hline MSF & & & & & & $0,96^{*}$ & $0,88^{*}$ & $0,97^{*}$ & $-0,88^{*}$ & $0,81^{*}$ & $-0,88^{*}$ & $-0,84^{*}$ & $0,95^{*}$ & $0,85^{*}$ & $-0,96^{*}$ & $0,99^{*}$ & $0,96^{*}$ \\
\hline MSC & & & & & & & $0,88^{*}$ & $0,98^{*}$ & $-0,80^{*}$ & $0,85^{*}$ & $-0,80^{*}$ & $-0,80^{*}$ & $0,93^{*}$ & $0,98^{*}$ & $-0,96^{*}$ & $0,95^{*}$ & $0,94^{*}$ \\
\hline MSR & & & & & & & & $0,82^{*}$ & $-0,81^{*}$ & $0,94^{*}$ & $-0,81^{*}$ & $-0,88^{*}$ & $0,93^{*}$ & $0,84^{*}$ & $-0,93^{*}$ & $0,88^{*}$ & $0,82^{*}$ \\
\hline IDQ & & & & & & & & & $-0,81^{*}$ & $0,78^{\text {ns }}$ & $-0,80^{*}$ & $-0,78^{\mathrm{ns}}$ & $0,91^{*}$ & $0,81^{*}$ & $-0,94^{*}$ & $0,96^{*}$ & $0,97^{*}$ \\
\hline E & & & & & & & & & & $-0,62^{\mathrm{ns}}$ & $0,99^{*}$ & $0,96^{*}$ & $-0,94^{*}$ & $-0,93^{*}$ & $0,91^{*}$ & $-0,84^{*}$ & $0,95^{*}$ \\
\hline A & & & & & & & & & & & $-0,91^{*}$ & $-0,73^{\mathrm{ns}}$ & $0,83^{*}$ & $0,76^{\mathrm{ns}}$ & $-0,83^{*}$ & $0,82^{*}$ & $0,65^{\mathrm{ns}}$ \\
\hline $\mathrm{Ci}$ & & & & & & & & & & & & $0,95^{*}$ & $-0,94^{*}$ & $-0,93^{*}$ & $0,91^{*}$ & $-0,84^{*}$ & $-0,91^{*}$ \\
\hline gs & & & & & & & & & & & & & $-0,96^{*}$ & $-0,97^{*}$ & $0,92^{*}$ & $-0,79^{*}$ & $-0,88^{*}$ \\
\hline ØPs2 & & & & & & & & & & & & & & $0,96^{*}$ & $-0,99^{*}$ & $0,92^{*}$ & $0,99^{*}$ \\
\hline ETR & & & & & & & & & & & & & & & $-0,95^{*}$ & $0,81^{*}$ & $0,99^{*}$ \\
\hline $\mathbf{N}$ & & & & & & & & & & & & & & & & $-0,93^{*}$ & $-0,98^{*}$ \\
\hline $\mathrm{AcN}$ & & & & & & & & & & & & & & & & & $0,93^{*}$ \\
\hline
\end{tabular}

Fonte: Autores (2020)

Em que: *significativo a $5 \%$ de probabilidade, ns não significativo. Altura - H, diâmetro - D, número de folhas -NF, área foliar - AF, área foliar especifica - AFE, massa seca foliar - MSF, massa seca caulinar - MSC, massa seca radicular MSR, índice de qualidade de Dickson - IQD, taxa transpiratória - $E$, taxa fotossintética - $A$, concentração interna de $\mathrm{CO}_{2}-\mathrm{Ci}$, condutância estomática - gs, eficiência do fotossistema II - ØPS2, taxa de transporte de elétrons - ETR, teor de nitrogênio da parte área - $\mathrm{N}$, acúmulo de $\mathrm{N}$ na parte aérea das mudas - $\mathrm{AcN}$ e eficiência do uso de $\mathrm{N}$ da parte aérea - EUN. 


\section{Conclusão}

Com base nos resultados obtidos neste estudo, é possível dizer que as mudas de Guazuma ulmifolia respondem a adubação nitrogenada, no entanto as doses entre 20 e $38,8 \mathrm{mg} \mathrm{dm}^{-3} \mathrm{de} \mathrm{N}$, de modo geral, foram as que promoveram os melhores resultados, tendo como fonte de $\mathrm{N}$ a ureia.

\section{Agradecimentos}

Somos gratos a Coordenação de Aperfeiçoamento de Pessoal de Nível Superior (CAPES), pela bolsa de doutorado e ao Instituto Federal Goiano - Campus Rio Verde pelo apoio e oportunidade.

\section{Referências}

AYRES, M. et al. BioEstat: aplicações estatísticas nas áreas das ciências biológicas e médicas. Belém: Sociedade Civil Mamirauá, 2007. 364 p.

BORGES, A. L.; SILVA, D. J. Fertilizantes para fertirrigação. In: SOUSA, V. F. et al. Irrigação e fertirrigação em fruteiras e hortaliças. Brasília: EMBRAPA Informação Tecnológica, 2011. p. 253-264.

CALIXTO JÚNIOR, J. T. et al. Phenolic composition and antiparasitic activity of plants from the Brazilian Northeast "Cerrado". Saudi Journal Biological Sciences, Arábia Saudita, v. 23, n. 3, p. 434-440, 2016.

CARLOS, L. et al. Growth and mineral nutrition in seedlings of jacarandá-da-bahia subjected to nutrient deprivation. Floresta, Curitiba, v. 45, n. 1, p. 107-116, 2015.

COLLI-SILVA, M. Guazuma in Flora do Brasil 2020 em construção. Rio de Janeiro: Jardim Botânico do Rio de Janeiro, 2019. Disponível em: http://floradobrasil.jbrj.gov.br/reflora/floradobrasil/FB9065. Acesso em: 07 ago. 2019.

DICKSON, A.; LEAF, A.; HOSNER, J. F. Quality appraisal of White spruce and white pine seedling stock in nurseries. Forestry Chronicle, Canadá, v. 36, n. 8, p. 10-13, 1960.

FERNANDES, M. C. O. C. et al. Crescimento e qualidade de mudas de Citharexylum myrianthum em resposta à fertilização nitrogenada. Advances in Forestry Science, Cuiabá, v. 6, n.1, p. 507-513, 2019.

FERREIRA, D. F. Sisvar: A Guide for Its Bootstrap Procedures in Multiple Comparisons. Ciência e Agrotecnologia, Lavras, v. 38, n. 2, p. 109-112, 2014.

FONSECA, E. P. et al. Padrão de qualidade de mudas de Trema micrantha (L.) Blume, produzidas sob diferentes períodos de sombreamento. Revista Árvore, Viçosa, MG, v. 26, n. 4, p. 515-523, 2002.

FREITAS, E. C. S. et al. Crescimento e qualidade de mudas de Cassia grandis Linnaeus f. em resposta à adubação fosfatada e calagem. Ciência Florestal, Santa Maria, v. 27, n. 2, p. 509-519, 2017.

GALINDO, F. S. et al. Manejo da adubação nitrogenada no capim-mombaça em função de fontes e doses de nitrogênio. Revista de Ciências Agrárias, Lisboa, v. 41, n. 4, p. 900-913, 2018.

GOMES, J. M. et al. Crescimento de mudas de Eucalyptus grandis em diferentes tamanhos de tubetes e fertilização N-P-K. Revista Árvore, Viçosa, MG, v. 27, n. 2, p. 113-127, 2003.

GONÇALVES, J. L. M. et al. Produção de mudas de espécies nativas: substrato, nutrição, sombreamento e fertilização. In: GONÇALVES, J. L. M.; BENEDETI, V. Nutrição e fertilização florestal. Piracicaba: IPEF, 2005. p. 309-350. 
GOUVEIA, P. A. R. Therapeutic Use Extract of Guazuma ulmifolia Lam of Northern Brazil. Journal of Microbiology \& Infectious Diseases, [s. l.]. v. 2, n. 3, p. 1-8, 2018.

MACEDO, L. Falta de exames, tratamento irregular, 'remédio caseiro': venezuelanos com HIV contam como enfrentam crise em seu país. G1, [s. l.], 23 maio 2019. Disponível em: https://g1.globo. $\mathrm{com} / \mathrm{mundo} /$ noticia/2019/05/23/falta-de-exames-tratamento-irregular-e-remedio-caseiroportadores-de-hiv-contam-como-enfrentam-a-crise-na-venezuela.ghtml. Acesso em: 12 fev. 2020.

MAGADLELA, A. et al. Low-phosphorus conditions affect the nitrogen nutrition and associated carbon costs of two legume tree species from a Mediterranean-type ecosystem. Australian Journal of Botany, Melbourne, v. 62, n. 1, p. 1-9, 2014.

MAO, Q. et al. Effects of simulated $\mathrm{N}$ deposition on foliar nutrient status, $\mathrm{N}$ metabolism and photosynthetic capacity of three dominant understory plant species in a mature tropical forest. Science of the Total Environment, Amsterdam, v. 610, n. 1, p. 555-562, 2018.

MORAES, G. P. et al. Adubação nitrogenada associada à inoculação com Azospirillum brasilense na cultura do milho. Agropecuária Técnica, Areia, v. 38, n. 3, p. 109-116, 2017.

NUNES, Y. R. F. et al. Germinação de sementes de Guazuma ulmifolia Lam. (Malvaceae) e Heteropterys byrsonimifolia A. Juss (Malpighiaceae) sob diferentes tratamentos de escarificação tegumentar. Unimontes Cientifica, Montes Claros, v. 8, n. 1, p. 43-52, 2006.

PORTADORES de HIV da Venezuela recorrem a folhas de árvore por falta de antirretrovirais. Folha de S. Paulo, São Paulo, 13 dez. 2018. Disponível em: https://www1.folha.uol.com.br/mundo/2018/12/ portadores-de-hiv-da-venezuela-recorrem-a-folhas-de-arvore-por-falta-de-antirretrovirais.shtml. Acesso em: 12 fev. 2020.

RAIJ, B. V. et al. Recomendações de adubação e calagem para o Estado de São Paulo. Campinas: Instituto Agronômico; Fundação IAC, 1997. 285 p.

RAJAN, J.; ANANDHAN S. V. Influence of nitrogen and potassium on root nutrient and root CEC of different tea cultivars (Camellia sinensis, C. assamica and C. assamica spp. Lasio calyx). Rhizosphere, [s. l.]. v. 1, n. 1, p. 36-44, 2016.

ROCHETTE, P. et al. Banding urea increased ammonia volatilization in a dry acidic soil. Journal of Environmental Quality, United States, v. 38, n. 4, p. 1383-1390, 2009.

SANTOS, H. G. et al. Sistema brasileiro de classificação de solos. Brasília: EMBRAPA, 2018. 356 p.

SIDDIQI, M. Y.; GLASS, A. D. M. Utilization index: a modified approach to the estimation and comparison of nutrient utilization efficiency in plants. Journal of Plant Nutrition, United States, v. 4, n. 3, p. 289-302, 1981.

SILVA, E. N. et al. Salt stress induced damages on the photosynthesis of physic nut young plants. Scientia Agricola, Piracicaba, v. 68, n. 1, p. 62-68, 2011.

SINGER, F. A condenação à morte dos pacientes de Aids na Venezuela. El Pais, Madrid, 07 set. 2018. Disponível em: https://brasil.elpais.com/brasil/2018/09/06/internacional/1536258399684413. html. Acesso em: 09 fev. 2020.

SOUZA, L. O guia completo da adubação nitrogenada para altas produtividades. [S. l.]: Instituto Agro, [2018]. Disponível em: https://institutoagro.com.br/adubacao-nitrogenada/. Acesso em: 02 dez. 2019.

TIAN, D. et al. Growth responses of trees and understory plants to nitrogen fertilization in a subtropical forest in China. Biogeosciences, Katlenburg-Lindau, v. 14, n. 14, p. 3461-3469, 2017.

WANG, Y. Y.; HSU, P. K.; TSAY, Y. F. Uptake, allocation and signaling of nitrate. Trends in Plant Science, Middletown, v.17, n. 8, p. 458-467, 2012. 